\title{
Integer-Forcing Linear Receivers: A Design Criterion for Full-Diversity STBCs
}

\author{
J. Harshan ${ }^{\dagger}$, Amin Sakzad ${ }^{\star}$, Emanuele Viterbo* \\ ${ }^{\dagger}$ Advanced Digital Sciences Center, Singapore, \\ ${ }^{\star}$ Clayton School of Information Technology, Monash University, Australia, \\ *Department of Electrical and Computer Systems, Monash University, Australia \\ Email: harshan.j@adsc.com.sg, amin.sakzad@monash.edu, emanuele.viterbo@monash.edu
}

\begin{abstract}
In multiple-input multiple-output (MIMO) fading channels, the design criterion for full-diversity space-time block codes (STBCs) is primarily determined by the decoding method at the receiver. Although constructions of STBCs have predominantly matched the maximum-likelihood (ML) decoder, design criteria and constructions of full-diversity STBCs have also been reported for low-complexity linear receivers. A new receiver architecture called Integer-Forcing (IF) linear receiver has been proposed to MIMO channels by Zhan et al. which showed promising results for the high-rate V-BLAST encoding scheme. In this work we address the design of full-diversity STBCs for IF linear receivers. We derive an upper bound on the probability of decoding error, and show that STBCs that satisfy the nonvanishing singular value (NVS) property provide full-diversity for the IF receiver. We also present simulation results to demonstrate that linear designs with NVS property provide full diversity for IF receiver. As a special case of our analysis on STBCs, we present an upper bound on the error probability for the VBLAST architecture presented by Zhan et al., and demonstrate that the IF linear receivers provide full receive diversity. Our results supplement the existing outage probability based results for the IF receiver.
\end{abstract}

\section{Introduction AND PRELIMINARIES}

Space-time coding is a powerful transmitter-side technique that assists reliable communication over multiple-input multiple-output (MIMO) fading channels. For a MIMO channel with $n_{t}$ transmit and $n_{r}$ receive antennas, a space-time block code (STBC) denoted by $\mathcal{C} \subset \mathbb{C}^{n_{t} \times T}$ is a finite set of complex matrices used to convey $\log _{2}(|\mathcal{C}|)$ information bits to the destination [1]. To recover the information bits all the observations collected across $n_{r}$ receive antennas and $T$ time slots at the destination are appropriately processed by a suitable decoder $\mathcal{D}$, e.g., maximum-likelihood (ML) decoder, zero-forcing (ZF) receiver, or a minimum mean square error (MMSE) receiver.

Considering the high computational complexity of the ML decoder, many research groups have addressed the design and construction of full-diversity STBCs that are matched to suboptimal linear receivers such as the ZF and the MMSE receivers [2]-[4]. These linear receivers reduce the complexity of the decoding process by trading off some error performance with respect to ML decoder. In [3] a new design criterion for full-diversity STBCs matched to ZF receivers is proposed, which imposes a constraint on the symbol-rate of STBCs. In particular, it has been proved that the symbol-rate of such STBCs is upper bounded by one. For some code constructions matched to ZF and MMSE linear receivers, we refer the reader to [2], [4]. In summary, a rate loss is associated with the design of full-diversity STBCs compliant to ZF and MMSE receivers [5]. A comparison of the decoding complexity, diversity, and the symbol-rate of STBCs for these decoders is summarized in Table 【 (given in next page).

A new receiver architecture called integer forcing (IF) linear receiver has been recently proposed [6] to attain higher rates with reduced decoding complexity. In such a framework, the source employs a layered transmission scheme and transmits independent codewords simultaneously across the layers. This has been referred to as the V-BLAST encoding scheme in [6]. At the receiver side, each layer is allowed to decode an integer linear combination of transmitted codewords, and then recover the information by solving a system of linear equations.

Although IF receivers are known to work well with the VBLAST scheme [7]-[12], not many works have investigated the suitability of IF receivers to decode STBCs. In [13] IF receiver has been applied to decode a layered transmission scheme involving perfect STBCs. Such an architecture has been shown to achieve the capacity of any Gaussian MIMO channel upto a gap that depends on the number of transmit antennas. An interesting question that arises from [13] is: What is the design criterion for full-diversity STBCs for IF linear receivers?

In order to answer the above question, it is paramount to characterize the structure of good STBCs for IF receivers. Towards that end, we study the error performance of IF receivers along the lines of [1], [3], and propose a design criterion for constructing full-diversity STBCs. The contributions of this paper is as follows: We study the application of IF linear receivers to decode STBCs in MIMO channels. We present a decoder error analysis for the IF receiver in order to obtain a design criterion for full-diversity STBCs. We show that STBCs that satisfy a criterion called the non-vanishing singular value (NVS) criterion provide full-diversity for the IF linear receiver. This is a stronger condition than the rank criterion [1] for the ML decoder. In [6] an outage probability based error analysis of the IF receiver is presented for the VBLAST scheme. As a special case of our error analysis on STBCs, we derive an upper bound on the error probability for 
TABLE I

COMPARISON OF STBCS FOR VARIOUS RECEIVERS: $n_{t}$ AND $n_{r}$ DENOTE THE NUMBER OF TRANSMIT AND RECEIVE ANTENNAS, RESPECTIVELY.

\begin{tabular}{|c|c|c|c|}
\hline Approach & $\begin{array}{c}\text { Decoding } \\
\text { Complexity }\end{array}$ & $\begin{array}{c}\text { Spatial } \\
\text { Diversity }\end{array}$ & $\begin{array}{c}\text { Symbol- } \\
\text { Rate }\end{array}$ \\
\hline ML & high & $n_{t} n_{r}$ & $\leq \min \left(n_{t}, n_{r}\right)$ \\
ZF \& MMSE & low & $n_{t} n_{r}$ & $\leq 1$ \\
IF & low & $n_{t} n_{r}$ & $\leq \min \left(n_{t}, n_{r}\right)$ \\
\hline
\end{tabular}

the architecture presented in [6] and show that the IF linear receivers provide full receive diversity for such a scheme. Thus, our results supplement the existing outage based results for the IF receiver.

The problem of constructing STBCs based on the minimum singular value criterion is not entirely new. In [14, Ch. 9] it has been shown that maximising the minimum singular value of the difference of codeword matrices provides the approximate universality property for STBCs in Multiple-Input SingleOutput (MISO) channels. It is to be noted that the criterion in [14] applies for the ML decoder. However, in this work, we show that the NVS criterion is applicable for the IF receiver for MIMO channels (not only MISO channels).

Notations. Boldface letters are used for vectors, and capital boldface letters for matrices. We let $\mathbb{R}, \mathbb{C}, \mathbb{Z}, \mathbb{Q}$, and $\mathbb{Z}[\imath]$ denote the set of real numbers, complex numbers, integers, rational numbers, and the Gaussian integers, respectively, where $\imath^{2}=-1$. We let $\mathbf{I}_{n}$ and $\mathbf{0}_{n}$ denote the $n \times n$ identity matrix and zero matrix and the operations $(\cdot)^{T}$ and $(\cdot)^{H}$ denote transposition and Hermitian transposition. We let $|\cdot|$ and $\|\cdot\|$ denote the absolute value of a complex number and the Euclidean norm of a vector, respectively. The operation $\mathbb{E}(\cdot)$ denotes mean of a random variable. We let $\lfloor x\rceil$ and $\lfloor\mathbf{v}\rceil$ denote the closest integer to $x$ and the component-wise equivalent operation. The symbol $\mathbf{X}_{j, m}$ denotes the element in the $j$-th row and $m$-th column of $\mathbf{X}$. For a matrix $\mathbf{X}$, the Frobenious norm $\sqrt{\sum_{j} \sum_{m}\left|\mathbf{X}_{j, m}\right|^{2}}$ is denoted by $\|\mathbf{X}\|_{F}$. The symbol $\mathcal{N}_{c}(0,1)$ denotes circularly complex Gaussian distribution with mean zero and unit variance. For an $n_{t} \times T$ matrix $\mathbf{X}$, the symbol $\sigma_{j}(\mathbf{X})$ denotes the $j$-th singular value of $\mathbf{X}$ for $1 \leq j \leq n_{t}$. The real and imaginary parts of a complex matrix $\mathbf{X}$ is denoted by $\operatorname{Re}(\mathbf{X})$ and $\operatorname{Im}(\mathbf{X})$, respectively. The symbol $\operatorname{Pr}(\cdot)$ denotes the probability operator.

\section{SySTEM MODEL}

The $n_{t} \times n_{r}$ MIMO channel consists of a source and a destination terminal equipped with $n_{t}$ and $n_{r}$ antennas, respectively. For $1 \leq i \leq n_{t}$ and $1 \leq j \leq n_{r}$, the channel between the $i$-th transmit antenna and the $j$-th receive antenna is assumed to be flat fading and denoted by the complex number $\mathbf{H}_{i, j}$. Each $\mathbf{H}_{i, j}$ remains constant for a block of $T$ $\left(T \geq n_{t}\right)$ complex channel uses and is assumed to take an independent realization in the next block. Statistically, we assume $\mathbf{H}_{i, j} \sim \mathcal{N}_{c}(0,1) \forall i, j$ across quasi-static intervals. The source conveys information to the destination through an

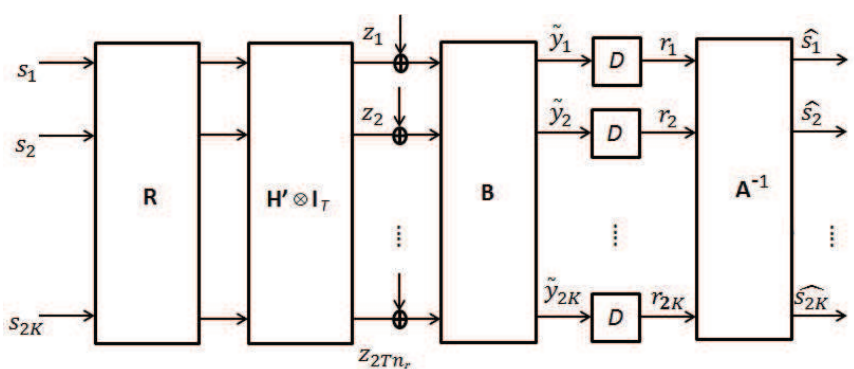

Fig. 1. IF linear receiver to decode STBCs where $\mathbf{R}$ is a code matrix obtained from vectorizing the components of the weight matrices $\left\{\mathbf{D}_{k}\right\}_{k=1}^{2 K}$ in $(1)$.

$n_{t} \times T$ STBC denoted by $\mathcal{C}$. We assume that a linear design

$$
\mathbf{X}_{\mathcal{L D}}\left(s_{1}, \ldots, s_{2 K}\right)=\sum_{k=1}^{2 K} \mathbf{D}_{k} s_{k}
$$

in $2 K$ real variables $\mathbf{s}=\left[\begin{array}{llll}s_{1} & s_{2} & \ldots & s_{2 K}\end{array}\right]^{T}$ is used to generate $\mathcal{C}$ by taking values from an underlying integer constellation $\mathcal{S} \subset \mathbb{Z}$. Here, the set $\left\{\mathbf{D}_{k} \in \mathbb{C}^{n_{t} \times T}\right\}_{k=1}^{2 K}$ contains the weight matrices of the design. Since we use the IF linear receiver to decode the STBC, we assume that $\mathcal{S}$ is a finite ring $\mathbb{Z}_{\sqrt{M}}=$ $\{0,1, \ldots, \sqrt{M}-1\}$ for some $M$, an even power of 2 . The symbols of $\mathcal{S}$ are appropriately shifted around the origin to reduce the transmit power, and subsequently reverted back at the receiver to retain the ring structure on $\mathcal{S}$. If $\mathbf{X}(\mathbf{s}) \in \mathcal{C}$ denotes a transmitted codeword matrix such that $\mathbb{E}\left[\left|\mathbf{X}_{i, t}\right|^{2}\right]=$ 1 for $1 \leq i \leq n_{t}, 1 \leq t \leq T$, then the received matrix $\mathbf{Y} \in \mathbb{C}^{n_{r} \times n_{t}}$ at the destination is given by

$$
\mathbf{Y}=\sqrt{\frac{P}{n_{t}}} \mathbf{H X}(\mathbf{s})+\mathbf{Z},
$$

where $\mathbf{H} \in \mathbb{C}^{n_{r} \times n_{t}}$ denotes the channel matrix, $\mathbf{Z} \in \mathbb{C}^{n_{r} \times T}$ denotes the AWGN with its entries that are i.i.d. as $\mathcal{N}_{c}(0,1)$. With this, the average receive signal power-to-noise ratio (SNR) per receive antenna is $P$. Throughout the paper, we assume a coherent MIMO channel where only the receiver has the complete knowledge of $\mathbf{H}$.

In the next subsection, we discuss the decoding procedure for STBCs based on the IF receiver.

\section{A. IF decoder for STBCs}

Since $\mathcal{C}$ is a linear dispersion code, the received matrix $\mathbf{Y}$ in (2) can be vectorized to obtain a noisy linear model as

$$
\mathbf{y}=\sqrt{\frac{P}{n_{t}}} \mathcal{H} \mathbf{s}+\mathbf{z}
$$

where $\mathcal{H} \in \mathbb{R}^{2 n_{r} T \times 2 K}$ is given by

$$
\mathcal{H}=\left(\mathbf{H}^{\prime} \otimes \mathbf{I}_{T}\right) \mathbf{R},
$$

such that

$$
\mathbf{H}^{\prime}=\left[\begin{array}{rr}
\operatorname{Re}(\mathbf{H}) & -\operatorname{Im}(\mathbf{H}) \\
\operatorname{Im}(\mathbf{H}) & \operatorname{Re}(\mathbf{H})
\end{array}\right] \in \mathbb{R}^{2 n_{r} \times 2 n_{t}},
$$

and $\mathbf{R} \in \mathbb{R}^{2 n_{t} T \times 2 K}$ is a code matrix obtained from vectorizing the components of the weight matrices $\left\{\mathbf{D}_{k}\right\}_{k=1}^{2 K}$. Here, the 
symbol $\otimes$ denotes the Kronecker product operator. After suitable scaling, (3) can be equivalently written (without changing the notation) as

$$
\mathbf{y}=\mathcal{H} \mathbf{s}+\sqrt{\frac{n_{t}}{P}} \mathbf{z} .
$$

To the linear model in (6), we apply the IF linear receiver as shown in Fig. 1 to recover $\hat{\mathbf{s}}$, a vector of decoded information symbols. We apply the constraint $2 K \leq 2 n_{r} T$ to avoid the case of under determined linear system. In order to decode an STBC using the IF receiver, the components of $\mathbf{s}$ are restricted to take values from a subset of integers such that $\left\{\mathcal{H} \mathbf{s} \mid \mathbf{s} \in \mathcal{S}^{2 K}\right\}$ is a lattice code carved from the lattice $\Lambda=\left\{\mathcal{H} \mathbf{s} \mid \mathbf{s} \in \mathbb{Z}^{2 K}\right\}$. This is the reason for choosing the components of $\mathbf{s}$ from the ring $\mathbb{Z}_{\sqrt{M}}$.

The goal of the IF receiver is to project $\mathcal{H}$ onto a nonsingular integer matrix $\mathbf{A} \in \mathbb{Z}^{2 K \times 2 K}$ by left multiplying $\mathcal{H}$ with a receiver filtering matrix $\mathbf{B} \in \mathbb{R}^{2 K \times 2 n_{r} T}$. After post processing by $\mathbf{B}$, we get

$$
\tilde{\mathbf{y}} \triangleq \mathbf{B y}=\mathbf{B} \mathcal{H} \mathbf{s}+\sqrt{\frac{n_{t}}{P}} \mathbf{B z} .
$$

The above signal model is applicable to all linear receivers including the ZF, MMSE (both cases $\mathbf{A}=\mathbf{I}_{2 K}$ ), and IF (where A is invertible over $\mathcal{S}$ ). For the IF receiver formulation, we write

$$
\tilde{\mathbf{y}}=\mathbf{A} \mathbf{s}+(\mathbf{B} \mathcal{H}-\mathbf{A}) \mathbf{s}+\sqrt{\frac{n_{t}}{P}} \mathbf{B z},
$$

where As is the desired signal component, and the effective noise is $(\mathbf{B} \mathcal{H}-\mathbf{A}) \mathbf{s}+\sqrt{\frac{n_{t}}{P}} \mathbf{B z}$. In particular, the effective noise power along the $m$-th row (henceforth referred to as the $m$-th layer) of $\tilde{\mathbf{y}}$ for $1 \leq m \leq 2 K$ is defined as

$$
g\left(\mathbf{a}_{m}, \mathbf{b}_{m}\right) \triangleq\left\|\mathbf{b}_{m} \mathcal{H}-\mathbf{a}_{m}\right\|^{2} \bar{E}+\frac{n_{t}}{2 P}\left\|\mathbf{b}_{m}\right\|^{2},
$$

where $\mathbf{a}_{m}$ and $\mathbf{b}_{m}$ denote the $m$-th row of $\mathbf{A}$ and $\mathbf{B}$, respectively, and $\bar{E}$ is the average energy of the constellation $\mathcal{S}$. A layer based model of the IF receiver architecture is as shown in Fig. 1. In order to reduce the effective noise power for each layer, the term $g\left(\mathbf{a}_{m}, \mathbf{b}_{m}\right)$ has to be minimized for each $m$ by appropriately selecting the matrices $\mathbf{A}$ and $\mathbf{B}$. For methods to select $\mathbf{A}$ and $\mathbf{B}$, we refer the reader to [6], [8]. In order to uniquely recover the information symbols, the matrix A must be invertible over the ring $\mathcal{S}$. In this work we are only interested in the STBC design for the IF receiver and hence, we assume that the optimal values of $\mathbf{A}$ and $\mathbf{B}$ are readily available.

We now present a procedure for decoding STBCs using the IF linear receiver. With reference to the signal model in Section II, the decoding procedure exploits the ring structure of the constellation $\mathcal{S}=\mathbb{Z}_{\sqrt{M}}=\{0,1, \ldots, \sqrt{M}-1\}$ with operations mod $\sqrt{M}$. The decoding procedure is as given below:

- Step 1 (Infinite lattice decoding over $\mathbb{Z}$ ): Each component of $\tilde{\mathbf{y}}$ is decoded to the nearest point in $\mathbb{Z}$ to get $\hat{\mathbf{y}}=\lfloor\tilde{\mathbf{y}}\rceil$, where $\lfloor\cdot\rceil$ denotes the round operation.
- Step 2 (Modulo operation onto $\mathcal{S}$ ): Perform the modulo $\sqrt{M}$ operation on the components of $\hat{\mathbf{y}}$ to obtain $\mathbf{r}=$ $(\hat{\mathbf{y}} \bmod \sqrt{M}) \in \mathcal{S}^{2 K}$.

- Step 3 (Solving system of linear equations): Solve the system of linear equations $\mathbf{r}=\mathbf{A} \hat{\mathbf{s}}$ over the ring $\mathbb{Z}_{\sqrt{M}}$. If A is invertible over the ring $\mathcal{S}$, then a unique solution is guaranteed. After solving the system of linear equations, information symbols are recovered from the components of $\hat{\mathbf{s}}$.

In the above decoding procedure, Step 2 and Step 3 are deterministic, while Step $\mathbf{1}$ involves recovering linear functions of the information symbols amidst noise. In the next section, we obtain an upper bound on the probability of error for Step 1, and then derive a design criterion for full-diversity STBCs.

\section{DESIGN CRITERION FOR STBCS}

We first present an upper bound on the probability of error for Step 1, i.e., decoding the $m$-th layer in the infinite lattice $\mathbb{Z}$ for $1 \leq m \leq 2 K$. The input to the decoder in Step 1 is

$$
\tilde{\mathbf{y}}_{m}=\mathbf{a}_{m} \mathbf{s}+\left(\mathbf{b}_{m} \mathcal{H}-\mathbf{a}_{m}\right) \mathbf{s}+\sqrt{\frac{n_{t}}{P}} \mathbf{b}_{m} \mathbf{z},
$$

where $\tilde{\mathbf{y}}_{m}$ denotes the $m$-th component of $\tilde{\mathbf{y}}$ and $\left(\mathbf{b}_{m} \mathcal{H}-\mathbf{a}_{m}\right) \mathbf{s}$ denotes the quantization noise term. For such a set-up, the effective noise power is given in (97. Note that the effective noise is not Gaussian distributed due to the quantization noise term. However, since the optimum value of $\mathbf{b}_{m}$ that minimizes (9) given $\mathbf{a}_{m}$ is

$$
\mathbf{b}_{m}=\mathbf{a}_{m} \mathcal{H}^{T}\left(\frac{n_{t}}{P \bar{E}} \mathbf{I}_{2 n_{r} T}+\mathcal{H} \mathcal{H}^{T}\right)^{-1}
$$

for large values of $P$, the above expression simplifies to $\mathbf{b}_{m}=$ $\mathbf{a}_{m} \mathcal{H}^{-1}$, where $\mathcal{H}^{-1}$ denotes the pseudo-inverse of $\mathcal{H}$. With this, for large values of $P$, the quantization noise term vanishes and the effective noise power is approximated by

$$
g\left(\mathbf{a}_{m}, \mathbf{b}_{m}\right)=\frac{n_{t}}{2 P}\left\|\mathbf{b}_{m}\right\|^{2} .
$$

Since we are interested in the full-diversity property of STBCs, which is a large SNR metric, we assume large values of $P$ in the probability of error analysis. Henceforth, we denote the probability of error for decoding the $m$-th layer in the infinite lattice $\mathbb{Z}$ by $P_{e}(m, \mathcal{H}, \mathbb{Z})$. The following Lemma provides an upper bound on $P_{e}(m, \mathcal{H}, \mathbb{Z})$.

Lemma 1: (Upper Bound on Probability of Error) For large values of $P$, the term $P_{e}(m, \mathcal{H}, \mathbb{Z})$ is upper bounded as

$$
P_{e}(m, \mathcal{H}, \mathbb{Z}) \leq \exp \left(-c P \epsilon_{1}^{2}(\Lambda)\right),
$$

where $c$ is some constant independent of $P$ and $\epsilon_{1}^{2}(\Lambda)$ is the minimum squared Euclidean distance of the lattice $\Lambda=$ $\left\{\mathbf{d} \mathcal{H}^{T} \mid \mathbf{d} \in \mathbb{Z}^{2 K}\right\}$.

Proof: Since the minimum Euclidean distance of $\mathbb{Z}$ is unity, an error in Step $\mathbf{1}$ is declared if $\sqrt{\frac{n_{t}}{P}}\left|\mathbf{b}_{m} \mathbf{z}\right| \geq \frac{1}{2}$. 
Therefore, we have

$$
P_{e}(m, \mathcal{H}, \mathbb{Z}) \triangleq \operatorname{Pr}\left(\sqrt{\frac{n_{t}}{P}}\left|\mathbf{b}_{m} \mathbf{z}\right| \geq \frac{1}{2}\right) .
$$

Since $\mathbf{b}_{m} \mathbf{z}$ is Gaussian distributed, using the Chernoff bound, $P_{e}(m, \mathcal{H}, \mathbb{Z})$ is bounded as

$$
\begin{aligned}
P_{e}(m, \mathcal{H}, \mathbb{Z}) & \leq \exp \left(-\frac{P}{4 n_{t}\left\|\mathbf{b}_{m}\right\|^{2}}\right) \\
& =\exp \left(-\frac{P}{4 n_{t}\left\|\mathbf{a}_{m} \mathcal{H}^{-1}\right\|^{2}}\right) .
\end{aligned}
$$

If $\mathbf{a}_{m}$ and $\mathbf{b}_{m}$ are chosen appropriately as in [6], then the upper bound

$$
\left\|\mathbf{a}_{m} \mathcal{H}^{-1}\right\|^{2} \leq \epsilon_{2 K}^{2}\left(\Lambda^{*}\right)
$$

holds good where $\epsilon_{2 K}^{2}\left(\Lambda^{*}\right)$ denotes the $2 K$-th successive minimum of the dual lattice

$$
\Lambda^{*}=\left\{\mathbf{d} \mathcal{H}^{-1} \mid \forall \mathbf{d} \in \mathbb{Z}^{2 K}\right\} .
$$

Here $\mathcal{H}^{-1}$ is a generator of the dual lattice $\Lambda^{*}$ of the lattice given by $\Lambda=\left\{\mathbf{d} \mathcal{H}^{T} \mid \forall \mathbf{d} \in \mathbb{Z}^{2 K}\right\}$, which is generated by the rows of $\mathcal{H}^{T}$. Thus we have the relation (see Lemma 4 in [6])

$$
\epsilon_{2 K}^{2}\left(\Lambda^{*}\right) \leq \frac{2 K^{3}+3 K^{2}}{\epsilon_{1}^{2}(\Lambda)},
$$

where $\epsilon_{1}^{2}(\Lambda)$ is the minimum squared Euclidean distance of the lattice $\Lambda$. Using the upper bounds of (13) and (14) in (12), the probability of error for decoding the $m$-th layer is upper bounded as

$$
P_{e}(m, \mathcal{H}, \mathbb{Z}) \leq \exp \left(-c P \epsilon_{1}^{2}(\Lambda)\right),
$$

where $c=\frac{1}{4 n_{t}\left(2 K^{3}+3 K^{2}\right)}$ is a constant. This completes the proof.

We now introduce a new property of linear designs to establish a relation between the upper bound in (15) and the structure of linear designs.

An infinite $\mathrm{STBC} \mathcal{C}_{\infty}$ generated from a linear design $\mathbf{X}_{\mathcal{L D}}$ in $2 K$ variables is given by

$$
\mathcal{C}_{\infty} \triangleq\left\{\mathbf{X}=\sum_{k=1}^{2 K} \mathbf{D}_{k} s_{k} \mid s_{k} \in \mathbb{Z} \forall k\right\} .
$$

As a special case, a finite $\mathrm{STBC} \mathcal{C}$ can be obtained from $\mathbf{X}_{\mathcal{L D}}$ by restricting the variables $s_{k}$ to $\mathcal{S}$ as

$$
\mathcal{C} \triangleq\left\{\mathbf{X}=\sum_{k=1}^{2 K} \mathbf{D}_{k} s_{k} \mid s_{k} \in \mathcal{S} \forall k\right\} .
$$

We let $\sigma_{\min }(\mathbf{X})=\min _{1 \leq j \leq n_{t}} \sigma_{j}(\mathbf{X})$ denote the minimum singular value of $\mathbf{X}$. With that, the minimum singular value of $\mathcal{C}_{\infty}$ is given by

$$
\sigma_{\min }\left(\mathcal{C}_{\infty}\right) \triangleq \inf _{\mathbf{X} \in \mathcal{C}_{\infty}, \mathbf{X} \neq \mathbf{0}} \sigma_{\min }(\mathbf{X}) .
$$

Using the above definition of the minimum singular value of the infinite code $\mathcal{C}_{\infty}$, we define a special class of linear designs as follows:
Definition 1: (Non-vanishing singular value property) A linear design $\mathbf{X}_{\mathcal{L D}}$ is said to have the non-vanishing singular value (NVS) property over $\mathbb{Z}$ if the corresponding infinite STBC $\mathcal{C}_{\infty}$ satisfies $\sigma_{\min }\left(\mathcal{C}_{\infty}\right) \neq 0$.

We now connect the NVS property of $\mathbf{X}_{\mathcal{L D}}$ and the fulldiversity property of $\mathcal{C}$ for the IF receiver in the following theorem.

Theorem 1: (Full-Diversity Design Criterion) If the linear design $\mathbf{X}_{\mathcal{L D}}$ has the NVS property, then any STBC $\mathcal{C}$ generated from $\mathbf{X}_{\mathcal{L D}}$ over $\mathcal{S}$ provides full-diversity with the IF linear receiver.

Proof: We write $\epsilon_{1}^{2}(\Lambda)$ in (15) as

$$
\epsilon_{1}^{2}(\Lambda)=\left\|\mathbf{d} \mathcal{H}^{T}\right\|^{2}=\left\|\mathcal{H} \mathbf{d}^{T}\right\|^{2}
$$

for some $\mathbf{d} \in \mathbb{Z}^{2 K}$. Note that the components of $\mathbf{d}$ that result in $\epsilon_{1}^{2}(\Lambda)$ need not be in $\mathcal{S}$. Further, $\epsilon_{1}^{2}(\Lambda)$ can be written as

$$
\epsilon_{1}^{2}(\Lambda)=\|\mathbf{H X}\|_{F}^{2}=\operatorname{Trace}\left(\mathbf{H U} \Sigma \mathbf{U}^{H} \mathbf{H}^{H}\right),
$$

where $\mathbf{X} \in \mathcal{C}_{\infty}, \mathbf{U} \Sigma \mathbf{U}^{H}$ is a singular value decomposition of $\mathbf{X X}^{H}$, and $\Sigma$ is the diagonal matrix comprising of the square of the singular values $\sigma_{j}(\mathbf{X})$ for $1 \leq j \leq n_{t}$. By denoting $\mathbf{H U}=\mathbf{G}$, we write

$$
\epsilon_{1}^{2}(\Lambda)=\operatorname{Trace}\left(\mathbf{G} \Sigma \mathbf{G}^{H}\right)=\sum_{j=1}^{n_{t}}\left\|\mathbf{g}_{j}\right\|^{2} \sigma_{j}^{2}(\mathbf{X}),
$$

where $\mathbf{g}_{j}$ is the $j$-th column of $\mathbf{G}$ and $\sigma_{j}(\mathbf{X})$ denotes the $j$-th singular value of $\mathbf{X}$, which is a function of the channel $\mathbf{H}$. If the STBC has the NVS property, then for any $\mathbf{d} \in \mathbb{Z}^{2 K}$ we apply $\sigma_{j}^{2}(\mathbf{X}) \geq \sigma_{\min }^{2}\left(\mathcal{C}_{\infty}\right) \forall j$, and hence,

$$
\epsilon_{1}^{2}(\Lambda) \geq \sum_{j=1}^{n_{t}}\left\|\mathbf{g}_{j}\right\|^{2} \sigma_{\min }^{2}\left(\mathcal{C}_{\infty}\right) .
$$

Using the above lower bound, we upper bound the expression in 15 as

$$
P_{e}(m, \mathcal{H}, \mathbb{Z}) \leq \exp \left(-c P \sigma_{\min }^{2}\left(\mathcal{C}_{\infty}\right) \sum_{j=1}^{n_{t}}\left\|\mathbf{g}_{j}\right\|^{2}\right) .
$$

Since $\mathbf{U}$ is a unitary matrix, the distribution of $\mathbf{G}$ is same as that of $\mathbf{H}$. Also, as $\sigma_{\min }^{2}\left(\mathcal{C}_{\infty}\right)$ is a constant and independent of $\mathcal{H}$, the random variables in the exponent are $\left\{\left\|\mathbf{g}_{j}\right\|^{2}\right\}$, which are chi-square distributed with degrees of freedom $2 n_{r}$. By averaging $P_{e}(m, \mathcal{H}, \mathbb{Z})$ over different realizations of $\left\|\mathbf{g}_{j}\right\|^{2}$, we obtain

$\mathbb{E}_{\mathcal{H}}\left[P_{e}(m, \mathcal{H}, \mathbb{Z})\right] \triangleq P_{e}(m, \mathbb{Z}) \leq\left(\frac{1}{1+c P \sigma_{\text {min }}^{2}\left(\mathcal{C}_{\infty}\right)}\right)^{n_{t} n_{r}}$

Since $P$ is dominant and $\sigma_{\min }\left(\mathcal{C}_{\infty}\right) \neq 0, P_{e}(m, \mathbb{Z})$ is upper bounded as

$$
P_{e}(m, \mathbb{Z})<\left(\frac{1}{c P \sigma_{\min }^{2}\left(\mathcal{C}_{\infty}\right)}\right)^{n_{r} n_{t}} .
$$

With the above result on the probability of error for each layer, we now setup an upper bound on the overall probability of error for Step 1. We declare an error in Step $\mathbf{1}$ if there is a 
decoding error in any one of the $2 K$ layers. Using the union bound, the overall probability of error is bounded as

$$
\operatorname{Pr}(\hat{\mathbf{y}} \neq \mathbf{A s} \mid \mathcal{H}) \leq \sum_{k=1}^{2 K} P_{e}(m, \mathcal{H}, \mathbb{Z})
$$

After taking expectation, the average probability of error for decoding Step 1 is

$$
\begin{aligned}
\operatorname{Pr}(\hat{\mathbf{y}} \neq \mathbf{A s}) & \triangleq \mathbb{E}_{\mathcal{H}}[\operatorname{Pr}(\hat{\mathbf{y}} \neq \mathbf{A s} \mid \mathcal{H})] \\
& \leq \sum_{k=1}^{2 K} \mathbb{E}_{\mathcal{H}}\left[P_{e}(m, \mathcal{H}, \mathbb{Z})\right] \\
& =\sum_{k=1}^{2 K} P_{e}(m, \mathbb{Z}) \\
& \leq \frac{c^{\prime}}{P^{n_{r} n_{t}}}
\end{aligned}
$$

where $c^{\prime}=\frac{2 K}{\left(c \sigma_{\min }^{2}\left(\mathcal{C}_{\infty}\right)\right)^{n_{t} n_{r}}}$. Notice that the upper bound in (16) is only a function of $\mathcal{C}_{\infty}$, and it is independent of the constellation $\mathcal{S}$. This shows that any STBC carved from a linear design with the NVS property provides diversity of $n_{t} n_{r}$ independent of the size of $\mathcal{S}$.

\section{Simulation Results on STBCS FOR IF ReCEIVERS}

Through simulation results, we show that a linear design with the NVS property provides full-diversity for the IF receiver. We use the Alamouti design given by

$$
\mathbf{X}_{A}=\left[\begin{array}{cc}
x_{1} & x_{2} \\
-x_{2}^{*} & x_{1}^{*}
\end{array}\right]
$$

to showcase the results. Using the structure of the above design, it can be shown that $\sigma_{\min }^{2}\left(\mathbf{X}_{A}\right)=\left|x_{1}\right|^{2}+\left|x_{2}\right|^{2}$. From the above expression, it is straightforward to observe that $\sigma_{\min }\left(\mathcal{C}_{\infty}\right)=1$ for $\mathbf{X}_{A}$. In Fig. 2, we present the bit error rate (BER) of the Alamouti code for the $2 \times 1$ MIMO channel when decoded with (i) the IF receiver, and (ii) the ML decoder. The plots confirm that the Alamouti code provides full-diversity with the IF receiver. For the simulation results, the method proposed in [8] is used throughout the paper to compute the $\mathbf{A}$ and $\mathbf{B}$ matrices for the IF receiver. It is well known that Alamouti code is ML decodable with lower computational complexity than the IF receiver. Despite its increased complexity, we have used the IF receiver for Alamouti code only to demonstrate that linear designs with the NVS property provide full diversity for the IF linear receiver.

\section{Diversity Results of IF RECEIVER FOR the V-BLAST ENCODING SCHEME}

In the seminal paper [6] on the IF receiver, a V-BLAST scheme is employed at the transmitter where the information symbols transmitted across different antennas are independent. In particular, the authors of [6] were interested in characterizing the diversity multiplexing trade-off (DMT) of the MIMO system with IF receiver. In order to derive the DMT, the system analysis in [6] is based on the outage probability. Hitherto, we

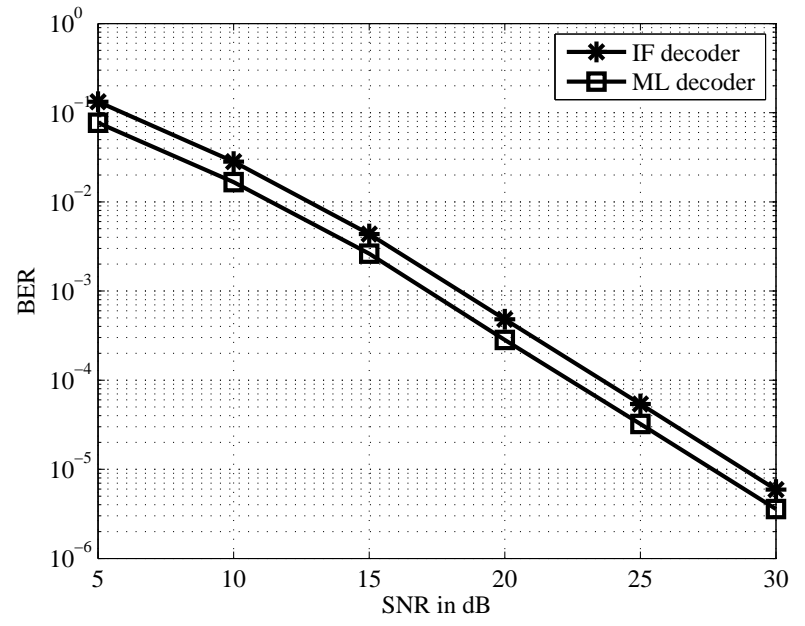

Fig. 2. BER comparison of Alamouti code with IF linear receiver and the ML decoder.

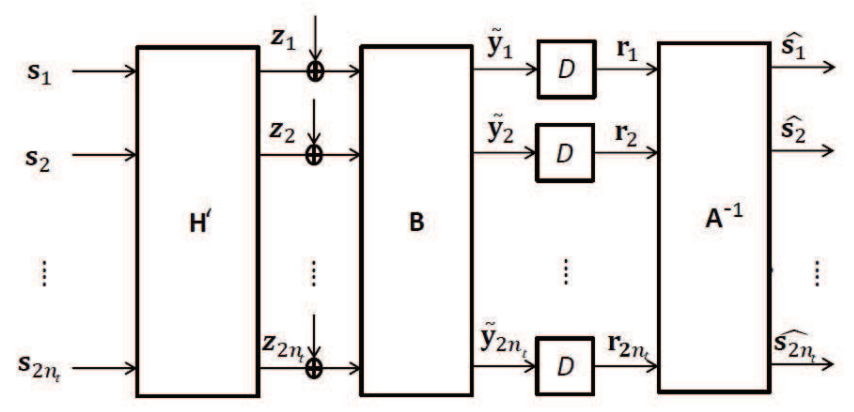

Fig. 3. IF receiver for the V-BLAST scheme where $\mathbf{H}^{\prime}$ is given in 5.

are not aware of any results that present the diversity results of the IF receiver based on the approach of [1], [3].

In this section we present an error probability analysis for the receiver architecture introduced in [6] shown in Fig. 3, and show that the IF linear receivers provide full receive diversity. This analysis is a special case of the analysis discussed in Section IIII We consider the uncoded case i.e, $T=1$ for the layered architecture in our analysis. With reference to the setting discussed in Section $\Pi$ the uncoded layered architecture corresponds to the use of a linear design

$$
\mathbf{X}_{\mathcal{L D}}\left(s_{1}, \ldots, s_{2 n_{t}}\right)=\sum_{k=1}^{2 n_{t}} \mathbf{D}_{k} s_{k}
$$

where $\mathbf{D}_{k} \in\left\{\mathbf{e}_{1}, \imath \mathbf{e}_{1}, \mathbf{e}_{2}, \imath \mathbf{e}_{2}, \ldots, \mathbf{e}_{n_{t}}, \imath \mathbf{e}_{n_{t}}\right\}$ such that $\left\{\mathbf{e}_{k}\right\}_{k=1}^{n_{t}}$ denotes the standard basis set in $\mathbb{R}^{n_{t}}$. Similar to Section [I, the received matrix $\mathbf{Y} \in \mathbb{C}^{n_{r} \times 1}$ in (2) can be vectorized to obtain a noisy linear model from $\mathbb{R}^{2 n_{t}}$ to $\mathbb{R}^{2 n_{r}}$ as

$$
\mathbf{y}=\sqrt{\frac{P}{n_{t}}} \mathcal{H} \mathbf{s}+\mathbf{z},
$$

where $\mathcal{H}$ becomes $\mathbf{H}^{\prime}$ after replacing $T=1$ and $\mathbf{R}=\mathbf{I}_{2 n_{t}}$ in (4). After applying the decoding procedure in Section П 
and following the analysis in Section [II, we upper bound the probability of decoding error for the $m$-th layer in Step $\mathbf{1}$ as

$$
P_{e}(m, \mathcal{H}, \mathbb{Z}) \leq \exp \left(-c P \epsilon_{1}^{2}(\Lambda)\right),
$$

where $1 \leq m \leq 2 n_{t}, c=\frac{1}{4 n_{t}\left(2 n_{t}^{3}+3 n_{t}^{2}\right)}$ is some constant and $\epsilon_{1}^{2}(\Lambda)$ is the minimum squared Euclidean distance of the lattice $\Lambda=\left\{\mathbf{d} \mathcal{H}^{T} \mid \forall \mathbf{d} \in \mathbb{Z}^{2 n_{t}}\right\}$.

Unlike Section [- where $n_{t} \times T$ dimensional matrices are codewords, in this case, $n_{t} \times 1$ dimensional complex vectors are codewords. We now discuss the singular value properties of these vectors to assist the proof for diversity order of the IF receiver. For any $\mathbf{x} \in \mathbb{Z}[\imath]^{n_{t}}$, let $\sigma_{1}(\mathbf{x})$ denote the non-zero singular value of $\mathbf{x}$. We define the minimum non-zero singular value of $\mathcal{C}_{\infty}=\mathbb{Z}[\imath]^{n_{t}}$ as $\sigma_{\min }\left(\mathcal{C}_{\infty}\right)=\inf _{\mathbf{x} \in \mathcal{C}_{\infty}, \mathbf{x} \neq \mathbf{0}} \sigma_{1}(\mathbf{x})$. We have the following result on $\sigma_{\min }\left(\mathcal{C}_{\infty}\right)$.

Lemma 2: For $\mathcal{C}_{\infty}=\mathbb{Z}[\imath]^{n_{t}}$, we have $\sigma_{\min }\left(\mathcal{C}_{\infty}\right) \geq 1$.

Proof: For any non-zero $\mathbf{x} \in \mathbb{Z}[\imath]^{n_{t}}$, the matrix $\mathbf{x x}^{H}$ has rank one, and hence, there is only one non-zero singular value of $\mathbf{x}$. This means we have $\sigma_{j}(\mathbf{x})=0$ for $j \neq 1$ and $\sigma_{1}(\mathbf{x}) \geq 0$ for any non-zero $\mathbf{x} \in \mathbb{Z}[\imath]^{n_{t}}$. Further, since the Trace property is preserved among similar matrices, we have $\sum_{j=1}^{n_{t}} \sigma_{j}(\mathbf{x})=\sigma_{1}(\mathbf{x})=\operatorname{Trace}\left(\mathbf{x x}^{H}\right)=\|\mathbf{x}\|^{2}$. Thus $\sigma_{1}(\mathbf{x}) \geq 1$ for any non-zero $\mathbf{x} \in \mathbb{Z}[\imath]^{n_{t}}$. This completes the proof.

Using the above Lemma, the diversity order of the IF receiver is derived in the following theorem.

Theorem 2: For the V-BLAST scheme, IF linear receiver provides full-receive diversity.

Proof: We can write $\epsilon_{1}^{2}(\Lambda)$ in (19) as

$$
\epsilon_{1}^{2}(\Lambda)=\left\|\mathbf{d} \mathcal{H}^{T}\right\|^{2}=\left\|\mathcal{H} \mathbf{d}^{T}\right\|^{2}
$$

for some $\mathbf{d} \in \mathbb{Z}^{2 n_{t}}$. Note that the components of $\mathbf{d}$ that result in $\epsilon_{1}^{2}(\Lambda)$ need not be in $\mathcal{S}$. Further, we can write $\epsilon_{1}^{2}(\Lambda)$ as

$$
\epsilon_{1}^{2}(\Lambda)=\|\mathbf{H} \mathbf{x}\|_{F}^{2}=\operatorname{Trace}\left(\mathbf{H} \mathbf{U} \Sigma \mathbf{U}^{H} \mathbf{H}^{H}\right),
$$

where $\mathbf{x} \in \mathcal{C}_{\infty}, \mathbf{U} \Sigma \mathbf{U}^{H}$ is a singular value decomposition of $\mathbf{x} \mathbf{x}^{H}$, and $\Sigma$ is the diagonal matrix containing the square of the singular values $\sigma_{j}(\mathbf{x})$ for $1 \leq j \leq n_{t}$. By denoting $\mathbf{H U}=\mathbf{G}$, we have

$$
\epsilon_{1}^{2}(\Lambda)=\operatorname{Trace}\left(\mathbf{G} \Sigma \mathbf{G}^{H}\right)=\sum_{j=1}^{n_{t}}\left\|\mathbf{g}_{j}\right\|^{2} \sigma_{j}^{2}(\mathbf{x}),
$$

where $\sigma_{j}(\mathbf{x})$ denotes the $j$-th singular value of $\mathbf{x}$, which is a function of the channel $\mathbf{H}$. Since $\mathbf{x}$ is a rank one vector, we have $\sigma_{j}(\mathbf{x})=0$ for $j \neq 1$ and from Lemma 2, we have $\sigma_{1}(\mathbf{x}) \geq 1$. Hence $\epsilon_{1}^{2}(\Lambda) \geq\left\|\mathbf{g}_{1}\right\|^{2}$ where $\mathbf{g}_{1}$ denotes the 1-st column of G. Using the above lower bound, we upper bound the expression in (19) as

$$
P_{e}(m, \mathcal{H}, \mathbb{Z}) \leq \exp \left(-c P\left\|\mathbf{g}_{1}\right\|^{2}\right) .
$$

By averaging $P_{e}(m, \mathcal{H}, \mathbb{Z})$ over different realizations of $\left\|\mathbf{g}_{1}\right\|^{2}$, we obtain

$$
\mathbb{E}_{\mathcal{H}}\left[P_{e}(m, \mathcal{H}, \mathbb{Z})\right] \triangleq P_{e}(m, \mathbb{Z}) \leq\left(\frac{1}{1+c P}\right)^{n_{r}}
$$

Thus the V-BLAST scheme in [6] provides full-receive diversity for IF linear receivers.

\section{Directions For Future Work}

An interesting direction for future work is to verify whether any known classes of high-rate STBCs (such as Golden code) provide full diversity with IF linear receivers. In such a case, the trade-off in the error performance can be studied between the low-complexity IF receivers and the high complexity ML decoder. Another direction of future work is to construct new STBC designs with large value of $\sigma_{\min }\left(\mathcal{C}_{\infty}\right)$ so that they perform well with IF receivers.

\section{ACKNOWLEDGEMENTS}

The authors would like to thank Roope Vehkalahti and Lakshmi Prasad Natarajan for their comments on the NVS property of STBCs. J. Harshan is supported by the HumanCentered Cyber-physical Systems Programme at the Advanced Digital Sciences Center from Singapore's Agency for Science, Technology and Research (A*STAR). Amin Sakzad and Emanuele Viterbo are supported by the Australian Research Council (ARC) under Discovery grants ARC DP150100285 and ARC DP160101077, respectively

\section{REFERENCES}

[1] V. Tarokh, N. Seshadri, and A. R. Calderbank, "Space-time codes for high data rate wireless communication: performance criterion and code construction," IEEE Trans. on Inform. Theory, vol. 44, pp. 744-765, Mar. 1998.

[2] J. K. Zhang, J. Liu, and K. M. Wong, "Linear Toeplitz space time block codes," in the Proc. of IEEE ISIT-05, Adelaide, Australia, Sept. 4-9, 2005, pp. 1942-1946.

[3] Y. Shang and Xiang-Gen Xia, "Space time block codes achieving full diversity with linear receivers," IEEE Trans. on Inform. Theory, vol. 54, no. 10 , pp. 4528-4547, Oct. 2008.

[4] H. Wang, Xiang-Gen Xia, Q. Yin, and B. Li "A family of space-time block codes achieving full diversity with linear receivers," IEEE Trans. on Communications, vol. 57, no. 12, pp. 3607-3617, Dec. 2009.

[5] A. H. Mehana and A. Nosratinia, "Diversity of MMSE MIMO receivers," IEEE International Symposium on Information Theory, Austin, TX, 2010, pp. 2163-2167.

[6] J. Zhan, B. Nazer, U. Erez, and M. Gastpar, "Integer-forcing linear receivers," IEEE ISIT, 2010, pp. 1022-1026, 2010.

[7] L. Wei and W. Chen, "Integer-forcing linear receiver design over MIMO channels," IEEE Global Communications Conference (GLOBECOM), 2012, Anaheim, CA, 2012, pp. 3560-3565.

[8] A. Sakzad, J. Harshan, and E. Viterbo, "Integer-forcing MIMO linear receivers based on lattice reduction," IEEE Trans. Wireless Communications, vol. 12, no. 10, pp. 4905-4915, Oct. 2013.

[9] A. Sakzad, J. Harshan, and E. Viterbo, "On complex LLL algorithm for integer forcing linear receivers," in the Proc. of IEEE Australian Communication Theory Workshop 2013, Adelaide, Australia, pp. 13-18, Jan. 2013.

[10] A. Sakzad and E. Viterbo, "Full Diversity Unitary Precoded IntegerForcing," in IEEE Trans. on Wireless Communications, vol. 14, no. 8, pp. 4316-4327, Aug. 2015.

[11] O. Ordentlich, U. Erez and B. Nazer, "Successive integer-forcing and its sum-rate optimality," 51st Annual Allerton Conference on Communication, Control, and Computing (Allerton), 2013, Monticello, IL, 2013, pp. 282-292.

[12] E. Domanovitz and U. Erez, "Performance of precoded integer-forcing for closed-loop MIMO multicast," IEEE Information Theory Workshop (ITW), 2014, Hobart, TAS, 2014, pp. 282-286.

[13] Or Ordentlich and Uri Erez, "Precoded integer-forcing universally achieves the MIMO capacity to within a constant gap," IEEE Information Theory Workshop (ITW), 2013, Sevilla, 2013, pp. 1-5.

[14] D. N. C. Tse and P. Viswanath, Fundamentals of Wireless Communications, 2005, Cambridge Univ. Press. 